\title{
Optimal conditions and the advantages of using laser microdissection and liquid chromatography tandem mass spectrometry for diagnosing renal amyloidosis
}

\author{
Michiko Aoki ${ }^{1} \cdot$ Dedong Kang $^{1} \cdot$ Akira Katayama $^{2} \cdot$ Naomi Kuwahara $^{1} \cdot$ Shinya Nagasaka ${ }^{1} \cdot$ Yoko Endo $^{1}$. \\ Mika Terasaki ${ }^{1}$. Shinobu Kunugi ${ }^{1}$ - Yasuhiro Terasaki ${ }^{1}$. Akira Shimizu ${ }^{1}$
}

Received: 23 November 2017 / Accepted: 4 January 2018 / Published online: 25 January 2018

(c) The Author(s) 2018. This article is an open access publication

\begin{abstract}
Background Liquid chromatography-tandem mass spectrometry (LC-MS/MS) has recently been utilized to accurately detect the amyloid proteins of renal amyloidosis. The present study investigated the optimal procedures for analyzing samples by LCMS/MS, and the advantage of using this technique to diagnosis renal amyloidosis.

Methods To detect amyloid proteins, laser microdissected glomeruli from AL $(n=13)$ or AA $(n=10)$ renal amyloidosis patients were digested and analyzed by LCMS/MS. To determine the best procedures for analyzing samples by LCMS/MS, we examined the suitability of tissue samples, frozen or formalin-fixed paraffin-embedded (FFPE), the number of dissected glomeruli required for analysis $(2,10$, or 50 glomeruli), and the amount of trypsin with or without dithiothreitol (DTT). We additionally compared the detection of amyloid proteins between immunostaining and LCMS/MS.

Results Examining 10 dissected glomeruli from FFPE sections digested with trypsin $3 \mu \mathrm{L}(0.1 \mathrm{mg} / \mathrm{mL})$ without DDT made it possible to detect amyloid protein in all $10 \mathrm{AA}$ and in 10 out of $12 \mathrm{AL}$ amyloidosis cases. All AA amyloidosis cases were diagnosed using immunohistochemistry for amyloid A. With immunostaining, however, there were several inconclusive immunoglobulin and/or their light chain staining noted in the AA or AL amyloidosis cases. Even so, LCMS/MS was able to accurately detect amyloid protein in renal amyloidosis.

Conclusion The use of 10 laser microdissected glomeruli $\left(170,000-220,000 \mu \mathrm{m}^{2}\right)$ with amyloid deposition from FFPE sections digested with trypsin $3 \mu \mathrm{L}(0.1 \mathrm{mg} / \mathrm{mL})$ allowed the accurate detection of amyloid protein in AA and AL amyloidosis.
\end{abstract}

Keywords Renal amyloidosis $\cdot$ Liquid chromatography tandem mass spectrometry · Amyloid protein ·

Immunofluorescence $\cdot$ Immunohistochemistry

\section{Introduction}

Amyloidosis is a disease caused by extracellular deposition of insoluble proteins with $\beta$-sheet structures exhibiting long fibrils in various organs [1]. The kidney is one of the most frequently involved organs. Amyloid deposits are generally identified by pathology on the basis of their applegreen birefringence under a polarized light microscope on

Akira Shimizu

ashimizu@nms.ac.jp

1 Department of Analytic Human Pathology, Nippon Medical School, 1-1-5, Sendagi, Bunkyo-ku, Tokyo 113-8602, Japan

2 Department of Biochemistry and Molecular Biology, Nippon Medical School, Tokyo, Japan
Congo Red-stained samples, with the presence of rigid nonbranching fibrils ranging from 7.5 to $10 \mathrm{~nm}$ in diameter seen on electron microscopy. Despite the similar morphologic findings observed for the amyloid depositions, there are at least 31 different amyloid proteins that are known to be causative proteins of amyloidosis. To design an appropriate therapeutic strategy, it is necessary to identify the true amyloid protein that is found in renal amyloidosis. Determination of the type of amyloid protein in amyloidosis has previously been identified by pathology that uses specific antibodies for amyloid protein in immunofluorescence (IF) and/or immunohistochemical (IHC) studies. However, it has been reported that, occasionally, these techniques cannot always detect true amyloid proteins, as non-specific positive or false negative stainings do occur [2-4]. Based on these issues, proteomic methods have recently been utilized 
to assess amyloid proteins. Sethi et al. [5, 6] demonstrated that proteomic methods can detect amyloid proteins using renal biopsy tissue samples. With this method, renal tissues with Congo Red-positive staining were dissected using a laser microdissection (LMD) technique for renal biopsy samples. After collection, the samples underwent further analysis using liquid chromatography-tandem mass spectrometry (LC-MS/MS).

Based on these previous studies, we have begun to investigate the detection of amyloid proteins using LCMS/MS proteomic analysis in renal amyloidosis. Our present study examined potential procedures that could be used to analyze renal amyloidosis samples by LCMS/MS. In addition, in $\mathrm{AL}$ and AA amyloidosis, we also examined the advantage of using LCMS/MS analysis compared to the classical IF and IHC methods.

\section{Materials and methods}

\section{Patients}

After a search of our departmental records for renal amyloidosis cases encountered between 1994 and 2016, we found a total of 23 cases that were diagnosed with AL (13 cases) and AA (10 cases) amyloidosis, including 6 autopsy cases: 4 cases of AL (AL5, AL6, AL8, and AL13) and 2 cases of AA (AA6 and AA8) amyloidosis. In our medical school hospital, the kidney samples in renal biopsies were fixed by $20 \%$ neutral formalin overnight, and the kidney samples in the autopsies were fixed by $20 \%$ formalin within 7 days. In control cases without amyloidosis, 5 renal biopsy cases of MCNS were used to compare between IF findings and the results of LCMS/ MS. In addition, 5 autopsy cases without amyloidosis and kidney diseases were used for the evaluation of the appropriate number of glomeruli for LCMS/MS analysis. Diagnoses of AA and AL amyloidosis in the present study were made based on the clinical information, laboratory data, and pathology. IF and IHC staining were performed using fluorescein isothiocyanate (FITC)-conjugated antibodies against IgG, IgM, and IgA (all from MBL, Nagoya, Japan), and polyclonal rabbit antibodies against human immunoglobulin $\kappa$ or $\lambda$ light chains (all from DakoCytomation, Glostrup, Denmark) and monoclonal mouse anti-human antibodies to amyloid A (DakoCytomation).

\section{Sample preparation and analysis using LCMS/MS}

\section{Tissue extraction}

For each case, $5-\mu \mathrm{m}$-thick sections of $20 \%$ neutral or $20 \%$ formalin-fixed paraffin-embedded (FFPE) or non-fixed frozen tissues were stained with Congo Red or Direct Fast Scarlet (DFS) on Leica PEN membrane glass slides. Glomeruli that were positive for the Congo Red stain were dissected by LMD using the Leica dissector (Leica LMD6000, Leica Microsystems, Mannheim, Germany). Before dissection of the glomeruli by LMD, the areas of glomerulus with Congo Red-positive staining and the areas of Bowman's capsule were measured by a computer-assisted image analyzer (Win Roof 2015 Version 3.1.0, Mitani Corp.,Tokyo, Japan), with the amyloid deposition rate calculated (Fig. 1).

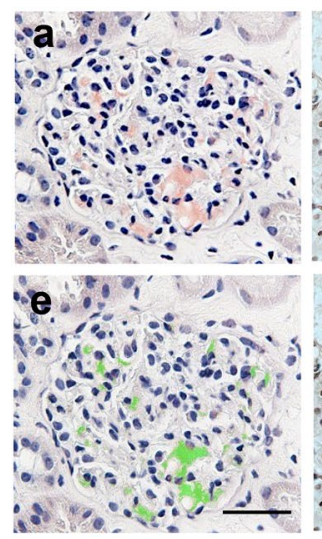

$6.2 \%$

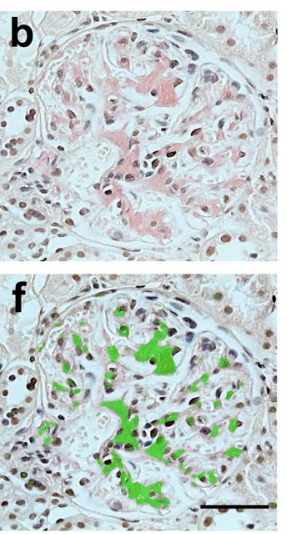

$11.4 \%$
Fig. 1 Percentage of amyloid deposition area in glomeruli. Before performing LMD of the glomeruli, the percentage of the amyloid deposition area in the region surrounded by Bowman's capsule was calculated as amyloid deposition rate (\%). Glomeruli with amyloid deposition in the Congo Red or Direct Fast Scarlet (DFS)-stained

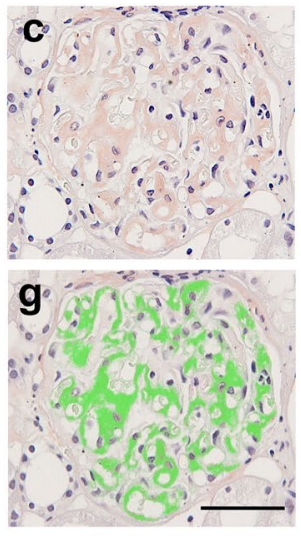

$28.8 \%$

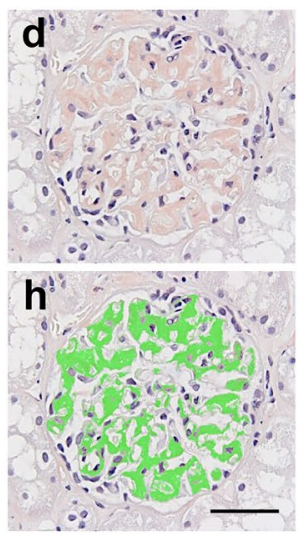

$41.4 \%$ sections were measured by a computer-assisted image analyzer (a-d, $\times 600$ ). Glomeruli with various amyloid deposition rates were noted in each glomerulus and in each of the renal amyloidosis cases (e-h, $\times 600)$. Bar $50 \mu \mathrm{m}$ 


\section{Tissue digestion}

Following LMD, microdissected tissue samples was collected into $0.5 \mathrm{~mL}$ microcentrifuge tube caps containing $40 \mu \mathrm{L}$ Tris/EDTA/0.002\% Zwittergent 3-16 buffer. After heating at $98{ }^{\circ} \mathrm{C}$ for $90 \mathrm{~min}$, and samples were sonicated at a frequency of $35 \mathrm{kHz}$ using a sonicator (Transsonication 890, Elma Schmidbauer GmbH, Singen, Germany) in a water bath for $90 \mathrm{~min}$. The microdissected tissue fragments were then digested into tryptic peptides overnight at $37^{\circ} \mathrm{C}$. Following the tryptic digestion of the samples, dithiothreitol (DTT) was added, and the samples were heated to $95^{\circ} \mathrm{C}$ for $5 \min [7]$.

\section{Mass spectrometry proteomic analysis}

After digestion of the tissue, all of the samples were analyzed by LCMS/MS using an amaZon ETD (Bruker Daltonics, Billerica, MA, USA). Raw data files from the LCMS/ MS were queried using two different algorithm databases (Mascot and X!Tandem). The results were combined and assigned peptide and protein probability scores according to the Scaffold database that was derived from the SwissProt human database (Proteome Software Inc., Portland, OR, USA). A list of proteins based on peptides identified by the mass spectrometry was generated for each case. Peptide identifications were accepted if they could be established at $>95 \%$ probability, with a 2 peptide minimum as specified by the peptide algorithm. The spectra value indicates the total number of mass spectra collected by LCMS/MS and matches the protein determined when using the proteomics software. A higher mass spectra number is typically indicative of greater abundance, thereby leading to a much better amino acid sequence coverage. Moreover, a higher number also demonstrates a higher confidence in the protein identification. It should be noted that when using the LCMS/MS and Scaffold database, a minimum of 4 mass spectra must be found in the samples to demonstrate any significant amyloid deposition $[5,6]$.

\section{Optimal condition required for LCMS/MS sample analysis}

To determine the appropriate number of glomeruli required for a successful LCMS/MS analysis, we investigated the use of 2, 10, and 50 laser microdissected glomeruli from FFPE tissues of 2 biopsy cases of AL amyloidosis and 9 autopsy cases, including 2 cases of AL and 2 cases of AA amyloidosis and 5 negative control cases of non-amyloidosis and nonkidney diseases. In addition, 10 dissected glomeruli from the FFPE tissues of $12 \mathrm{AL}$ amyloidosis and $10 \mathrm{AA}$ amyloidosis cases were digested and analyzed by LCMS/MS. We then examined the correlation between the amyloid deposition rate (percentage of Congo Red- or DFS-positive glomerular areas relative to the areas surrounded by Bowman's capsule) and the mass spectra values for the amyloid proteins determined by the LCMS/MS analysis. Furthermore, we additionally evaluated the appropriate amount of trypsin and the necessity of adding a reducing agent such as DTT (Wako, Tokyo, Japan) during the digestion of amyloid protein into peptides. The dissected 10 glomeruli with amyloid deposition from the FFPE tissue sections were digested by 3, 6,12, and $20 \mu \mathrm{L}$ of trypsin $(0.1 \mathrm{mg} / \mathrm{mL})$ (Promega, Madison, WI, USA) with or without $2 \mu \mathrm{L}$ of DTT (100 mM).

\section{Findings of the IF and IHC studies and LCMS/MS analysis}

To evaluate the advantage of using LCMS/MS analysis versus the classical pathology techniques for the detection of amyloid proteins in AA and AL amyloidosis patients, we compared the findings of IF and IHC for the immunoglobulins and amyloid proteins with the findings for the LCMS/ MS analysis.

\section{Results}

\section{Optimal conditions required for LCMS/MS sample analysis}

Renal glomeruli with amyloid deposition were microdissected using the LMD technique, and samples were digested and evaluated by LCMS/MS. This part of the study examined the optimal conditions required to successfully detect amyloid protein by LCMS/MS. After measurement of the percentage of the amyloid deposition rate found within the glomeruli (Fig. 1), we investigated the appropriate number of glomeruli required to successfully perform the LCMS/MS analysis using biopsy and autopsy cases. To determine this, we examined the degree of the amyloid protein spectra in 2, $10\left(170,000\right.$ to $\left.220,000 \mu^{2}\right)$, and 50 dissected glomeruli from FFPE samples obtained from $4 \mathrm{AL}$ amyloidosis and 2 AA amyloidosis cases as well as 5 control autopsy cases without amyloidosis and kidney diseases (Fig. 2a). The spectra numbers for the amyloid protein increased in accordance with the number of dissected glomeruli from 2 to 50 in both the $\mathrm{AL}$ and $\mathrm{AA}$ amyloidosis cases. In control cases without amyloidosis and kidney diseases, the amyloid protein such as immunoglobulin light chain or serum amyloid A could not be detected even in the samples of 50 dissected glomeruli, although the spectra numbers for cytoskeletal proteins such as keratin, actin, and vimentin increased in accordance with the number of dissected glomeruli from 2 to 50. Importantly, all of the samples having more than 10 glomeruli were shown to have more than 4 mass spectra, 

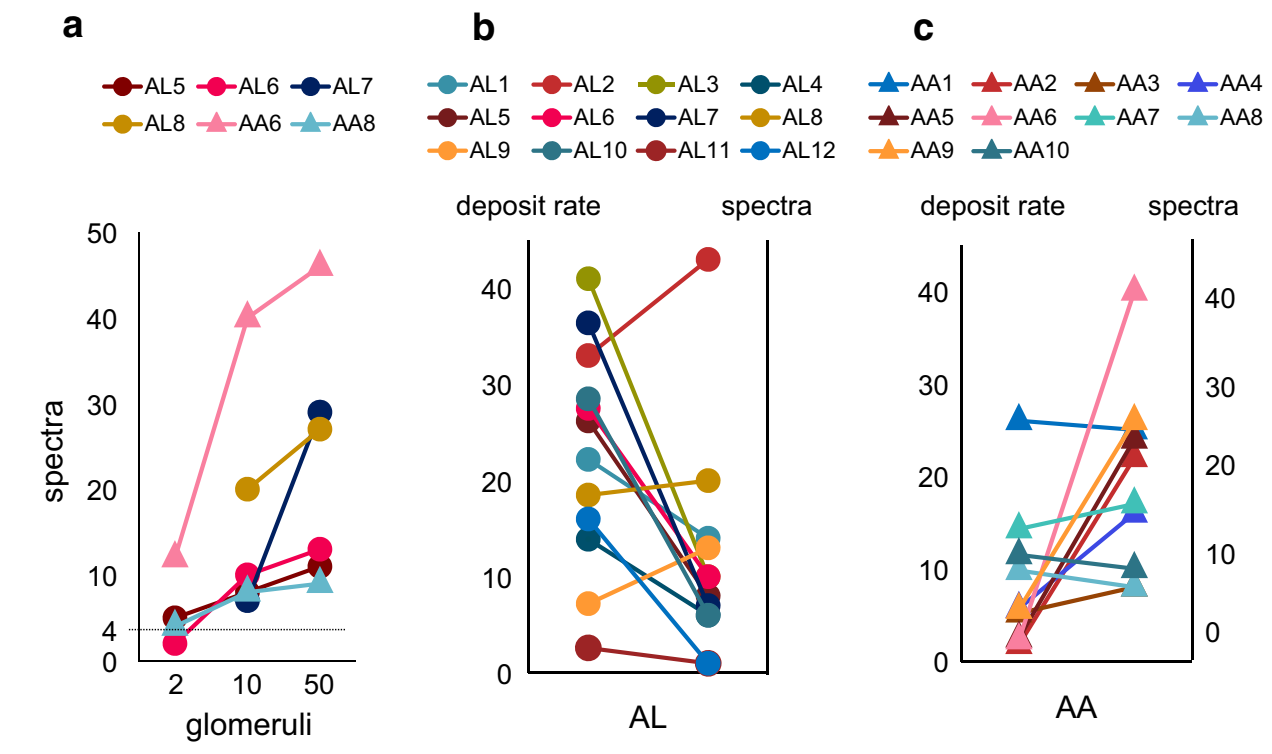

$A L$

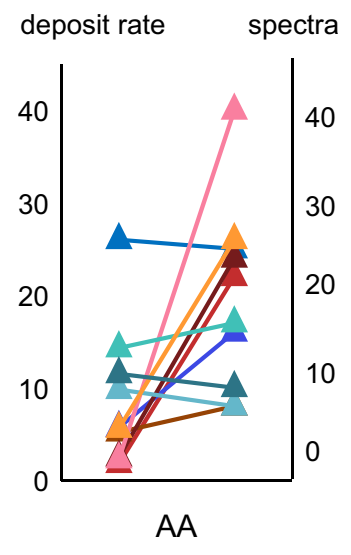

Fig. 2 The protein probability score spectra numbers for the amyloid proteins in the LCMS/MS analysis. a In both the AL and AA amyloidosis cases, the spectra numbers for the immunoglobulin $\kappa$ or $\lambda$ chain or the serum amyloid A in accordance with the number of glomeruli dissected (i.e., 2, 10, and 50). In all 6 cases, the spectra number for amyloid protein ( $\kappa$ or $\lambda$ chain or serum amyloid $\mathrm{A}$ ) increased above the minimum spectra number of 4 when 10 glomeruli were dissected. b When using 10 dissected glomeruli, the number of spectra for the immunoglobulin $\kappa$ or $\lambda$ chain was greater than 4 in 10 out of 12 cases

which is considered to be significant for amyloid protein in amyloidosis cases. However, the samples containing only 2 glomeruli were shown to have fewer than 4 spectra in 1 out of 4 cases.

Subsequently, we then examined the correlation between the amyloid deposition rate in the glomeruli and the mass spectra numbers for the amyloid protein in the AL and AA amyloidosis cases (Fig. 2b, c). The amyloid deposition rate in the glomeruli was $22.7 \pm 11.6 \%$, while the mean spectra number for the immunoglobulin light chains from the 10 dissected glomeruli was $11.6 \pm 11.2$ in the 12 AL amyloidosis cases. However, in the groups with 10 dissected glomeruli, there were 2 out of the $12 \mathrm{AL}$ amyloidosis cases for which the mass spectra exhibited less than 4 spectra (AL11 case: $2.6 \%$ in amyloid deposition rate; and AL12 case: $16.0 \%$ in amyloid deposition rate). In the $10 \mathrm{AA}$ amyloidosis cases, the amyloid deposition rate in the glomeruli was $11.1 \pm 8.6 \%$, while the mean spectra number for the serum amyloid A from the 10 dissected glomeruli was 19.6 \pm 9.9 .

After digestion of the ten dissected glomeruli from the FFPE samples, we evaluated the appropriate amount of trypsin and DTT required for the proteolysis in three cases of each in the AL and AA amyloidosis groups (Fig.3). The 10 dissected glomeruli were digested by $3,6,12$, and $20 \mu \mathrm{L}$ of trypsin $(0.1 \mathrm{mg} / \mathrm{mL})$ with or without DTT. For the AL (exceptions were cases AL11 and AL12). $\mathbf{c}$ The number of spectra for the serum amyloid A was greater than 4 in all $10 \mathrm{AA}$ amyloidosis cases when using 10 dissected glomeruli. The spectra numbers for the amyloid proteins in both the AL and AA amyloidosis cases were not clearly correlated with the degree of the percentage of amyloid deposition in the glomeruli (amyloid deposition rate). The mean spectra for AA amyloidosis was larger than that found for AL amyloidosis, though the amyloid deposition rate was smaller in AA amyloidosis versus AL amyloidosis

amyloidosis samples, the spectra numbers for the immunoglobulin light chain did not increase, though the amount of trypsin was increased or DTT was added. The highest spectra number was found for the sample with $3 \mu \mathrm{L}(0.1 \mathrm{mg} /$ $\mathrm{mL}$ ) trypsin. For AA amyloidosis, the spectra number for the serum amyloid A was the highest in the sample with $6 \mu \mathrm{L}(0.1 \mathrm{mg} / \mathrm{mL})$ trypsin in 2 out of 3 cases, while it was highest for the $3 \mu \mathrm{L}(0.1 \mathrm{mg} / \mathrm{mL})$ trypsin with DTT in 1 out of 3 cases. Similar to that seen for the digestion for AL amyloidosis, the spectra number for all of the AA amyloidosis cases exhibited more than four spectra of serum amyloid A in the samples with $3 \mu \mathrm{L}(0.1 \mathrm{mg} / \mathrm{mL})$ trypsin.

\section{Findings of the IF and IHC studies and the LCMS/MS analysis}

In all of the AA amyloidosis cases, IHC evaluations of amyloid A demonstrated clearly positive amyloid deposition areas (Fig. 4). However, we also observed equivocal and irregular weak positive staining against immunoglobulins in all of these AA amyloidosis cases. The LCMS/ MS analysis of the AA amyloidosis cases examined both frozen and FFPE samples (Fig. 5). For the tissues from the FFPE samples, the analysis demonstrated that the amyloid protein (serum amyloid A) could be detected as 


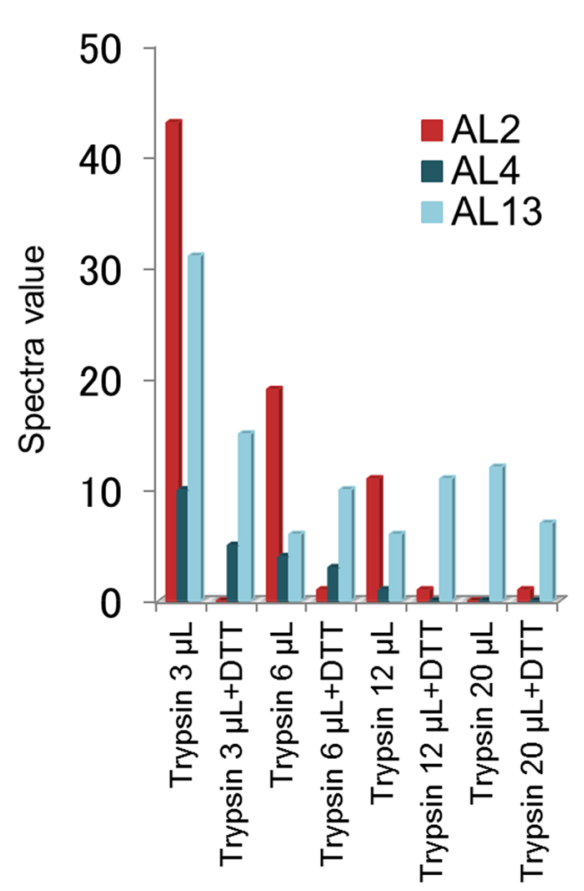

Fig. 3 Condition used for digestion of the 10 glomeruli dissected from formalin-fixed paraffin-embedded(FFPE) sections. a After digestion of the 10 glomeruli dissected from the FFPE tissue sections of AL amyloidosis patients, the spectra numbers for the immunoglobulin light chain were the highest in samples added with $3 \mu \mathrm{L}$ of trypsin without DTT. b The spectra numbers for the serum amyloid A

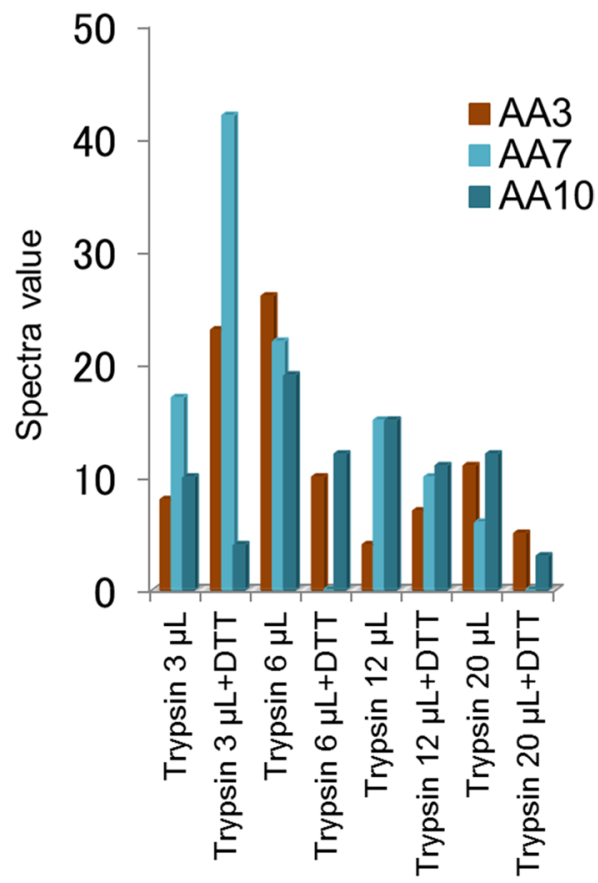

were highest in the samples with $6 \mu \mathrm{L}$ of trypsin in AA3 and AA10, and in the sample with $3 \mu \mathrm{L}$ of trypsin and DTT in AA7. The sample with $3 \mu \mathrm{L}$ of trypsin without DTT, which was the optimal condition for digestion of the AL amyloid, exhibited a sufficient spectra number above 4 that allowed for the diagnosis of amyloidosis in all 3 of the AA amyloidosis cases
Fig. 4 Immunofluorescence (IF) and immunohistochemistry (IHC) findings in four representative AA amyloidosis cases. The IF study in the four representative AA amyloidosis cases (AA1 to AA4 case) exhibited several degrees of positive stains for $\operatorname{IgM}, \operatorname{IgG}$, and/or $\operatorname{IgA}(\times 600)$. The IHC evaluation showed that all of the AA amyloidosis cases were positive for serum amyloid A $(\times 600)$. Bar 50 $\mu \mathrm{m}$

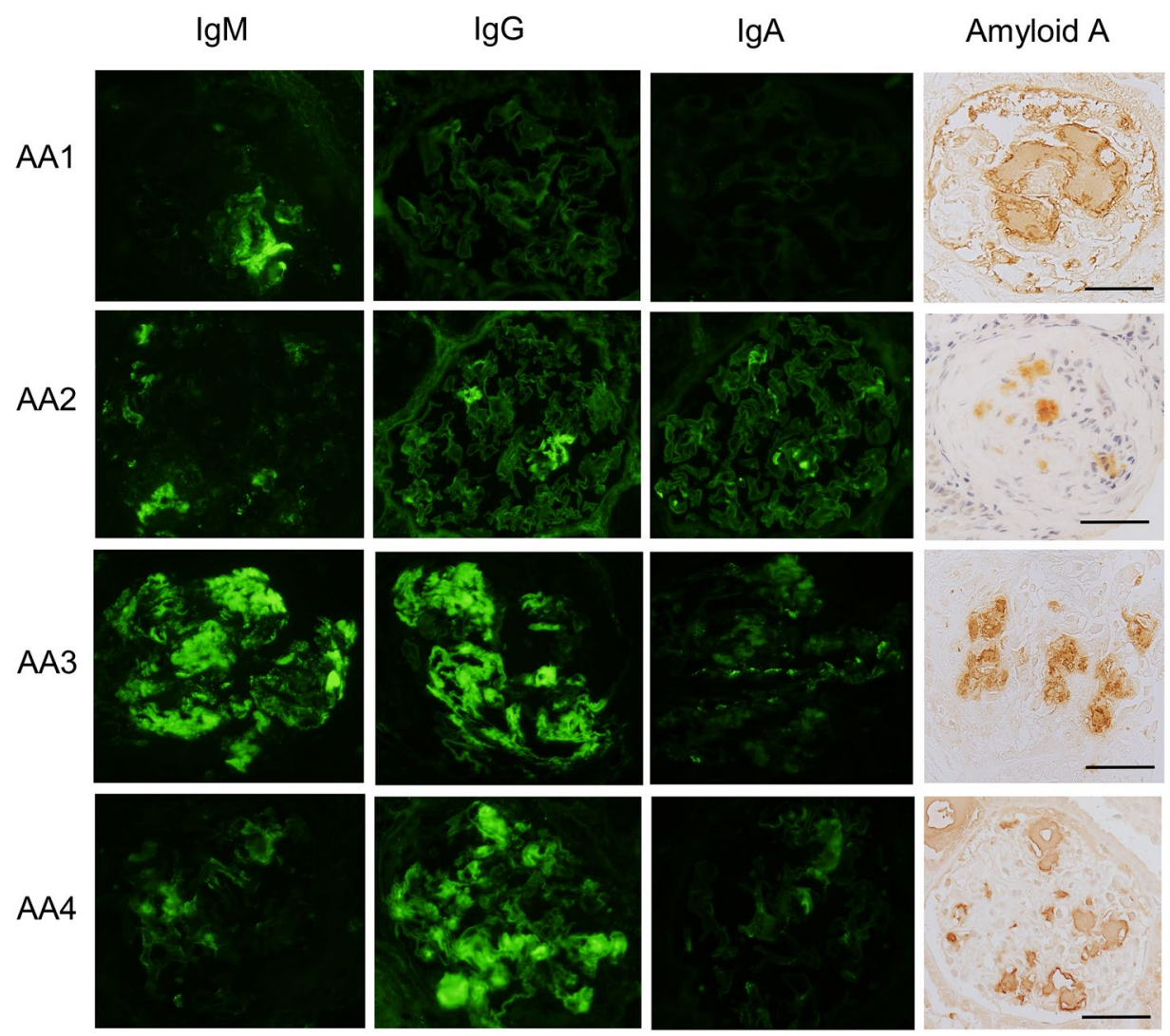




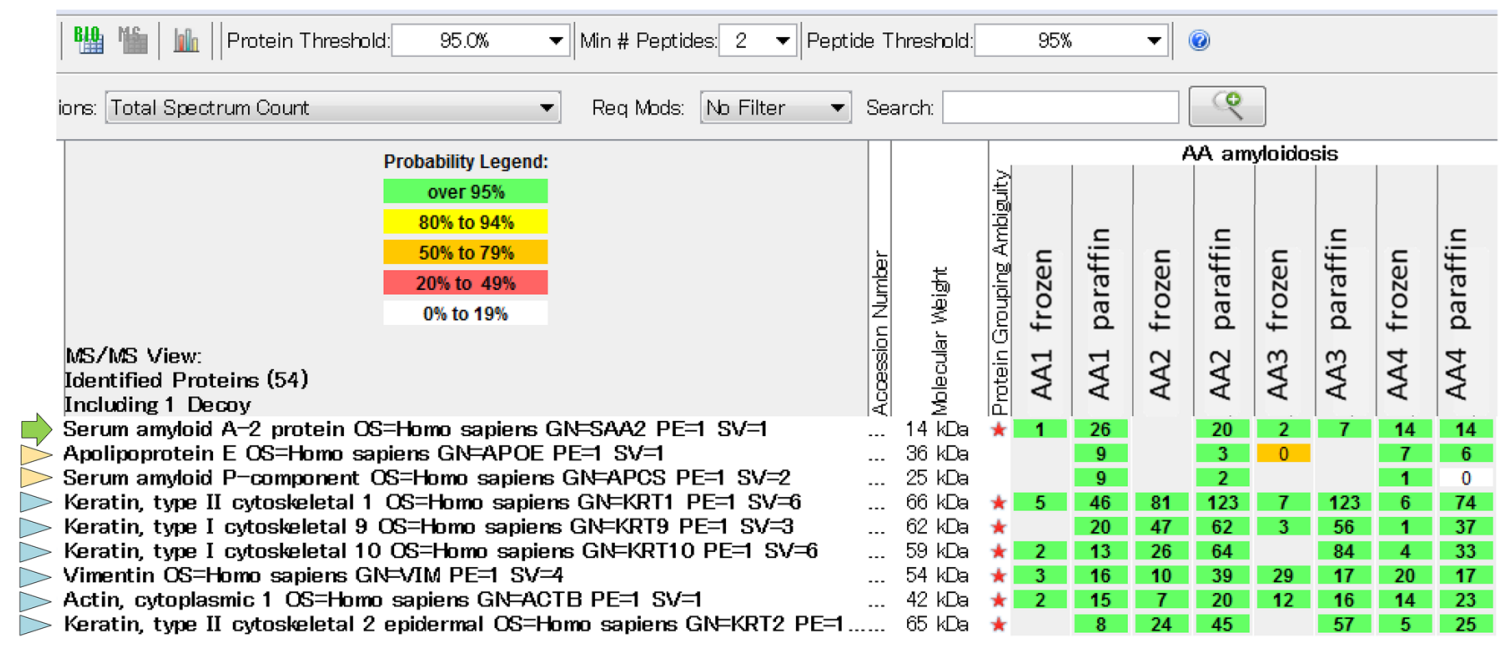

Fig. 5 Results of LCMS/MS according to the Scaffold database for AA amyloidosis. LCMS/MS analysis results showed that the serum amyloid A (green color arrow) was the most commonly identified protein, in the FFPE sections in the AA1-AA4 cases, with protein probability spectra number scores of $25,22,8$, and 16 (highlighted in green color), respectively. Cases AA1-AA4 were the same cases shown in the IF and IHC findings in Fig. 4. The spectra numbers for

high spectra numbers. In contrast, for the serum amyloid $\mathrm{A}$ in the frozen tissues, there were no or very low spectra numbers found. In addition, the spectra numbers for the apolipoprotein E (ApoE) and serum amyloid P component (SAP), which are known to be major constituents of amyloid and referred to as "amyloidogenic proteins", were also found to have no or low spectra in the samples from the frozen tissues. ApoE and SAP were detected in almost all cases as high spectra numbers in the samples from the FFPE tissue. The spectra numbers of cytoskeletal proteins such as keratin, actin, and vimentin were also higher in FFPE samples than those in frozen samples. Furthermore, in the AA amyloidosis cases, LCMS/MS could not detect any of the immunoglobulins that were observed in the IF staining.

The IF and IHC evaluations of AL amyloidosis showed that there were inconclusive positive staining and/or false negative staining against immunoglobulins and/or immunoglobulin light chains detected in the glomeruli with amyloid deposition (Fig. 6). However, the LCMS/MS analysis of the 10 dissected glomeruli from FFPE tissues showed that only a single immunoglobulin light chain with more than 4 spectra could be detected (Fig. 7). The single immunoglobulin light chain detected in these cases was the same as the light chain found during the analysis of the serum by IEP. ApoE and SAP were detected by LCMS/MS with more than four spectra in all of the AL amyloidosis cases. It should be noted that, in the one case, (AL2), where the M-protein could not be observed in the serum or urine by IEP, the LCMS/MS analysis demonstrated that there was deposition of the $\kappa$ light the serum amyloid A and amyloidogenic proteins (apolipoprotein E and serum amyloid P: orange color arrowhead) were higher in FFPE sections than those in frozen samples. There were no spectra detected for the immunoglobulins in either the frozen or the FFPE specimens for the LCMS/MS analysis. The spectra numbers for cytoskeletal proteins such as keratin, actin, and vimentin (blue color arrowheads) were higher in FFPE tissues than in frozen tissues

chain in glomeruli, which suggests that LCMS/MS may be a more highly sensitive method as compared to IEP.

To compare the IF findings and the results of LCMS/ MS in the control cases without amyloidosis, we analyzed 5 biopsy cases of MCNS. The LCMS/MS analysis of these cases did not detect any amyloid proteins such as the immunoglobulin light chain or serum amyloid $\mathrm{P}$, as well as amyloidogenic proteins such as ApoE and SAP. Furthermore, although irregular positive staining of IgM was noted in the glomeruli in our cases, LC-MS/MS analysis could not detect any immunoglobulin heavy chains.

\section{Discussion}

The LCMS/MS technique is a combination of liquid chromatography (LC) with mass spectrometry (MS), while MS/ MS is the combination of two mass analyzers in one mass spectrometry instrument. Therefore, LCMS/MS can identify a protein with high specificity and sensitivity. As a result, this new technique has been used to accurately diagnose the type of renal amyloidosis [5, 6]. This study attempted to determine the optimal conditions required for LCMS/MS sample analyses. We first examined whether FFPE or frozen tissues were better for the detection of amyloid protein, and then determined how many glomeruli with amyloid deposition were necessary to have on the $5-\mu \mathrm{m}$-thick sections. Other laboratories have used LMD to dissect 2 to 4 glomeruli from 10- $\mu \mathrm{m}$-thick sections of FFPE tissues, with each of the samples containing an area of 50,000-60,000 $\mu \mathrm{m}^{2}$ 
Fig. 6 Immunofluorescence (IF), immunohistochemistry (IHC), and immunoelectrophoresis (IEP) findings in 4 representative AL amyloidosis cases. Equivocal positive or false negative staining was observed for the IF and IHC evaluations for immunoglobulins and their light chains in most of the AL amyloidosis cases, with the exception of the AL1 case $(\times 600)$. In the AL1 case, a positive $\kappa$ light chain was found by the IF and IEP study. The IF or IHC results did not match the IEP results in the other 3 cases (AL2, AL3, and AL4). This highlights the difficulties in trying to accurately diagnose the type of AL amyloidosis based on only the IF findings. Bar $50 \mu \mathrm{m}$

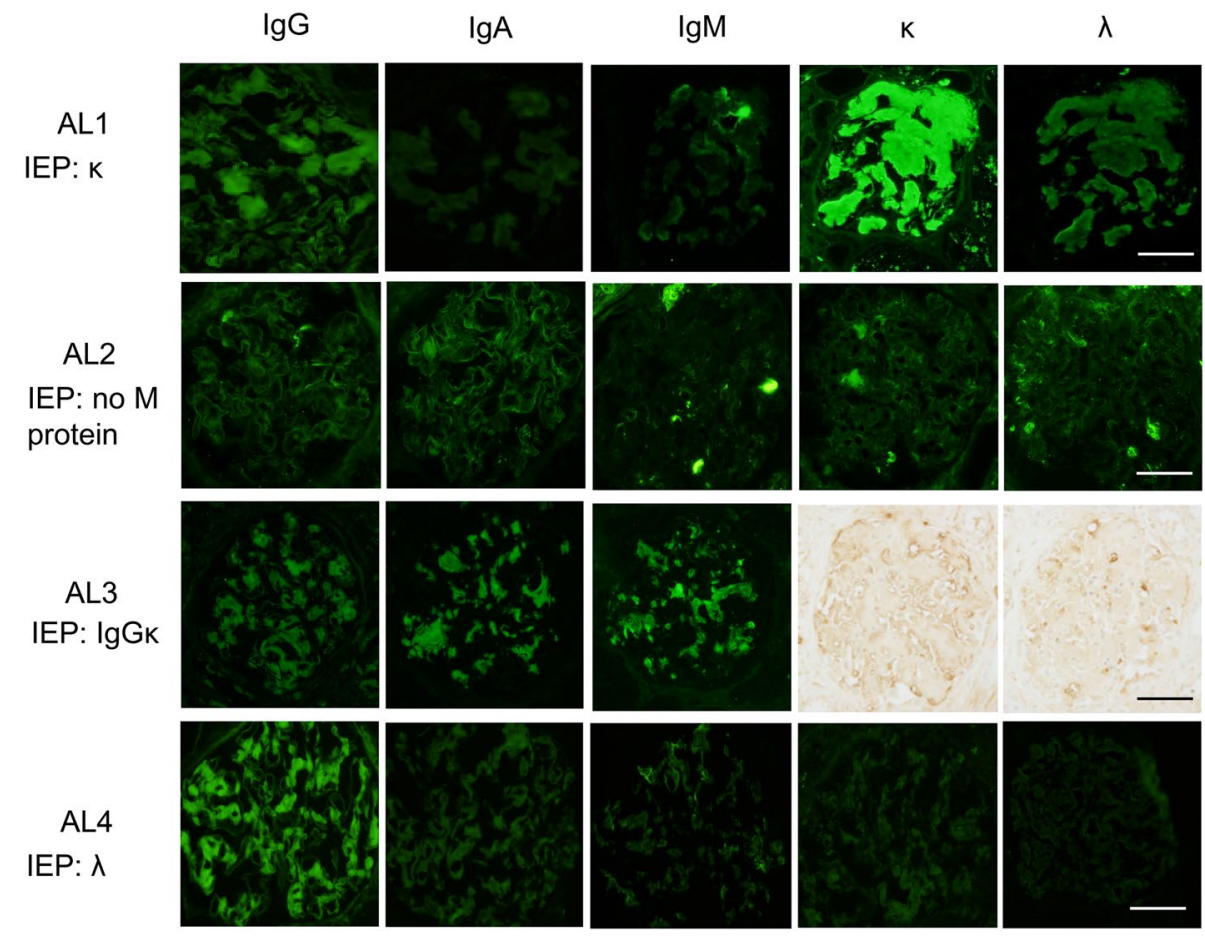

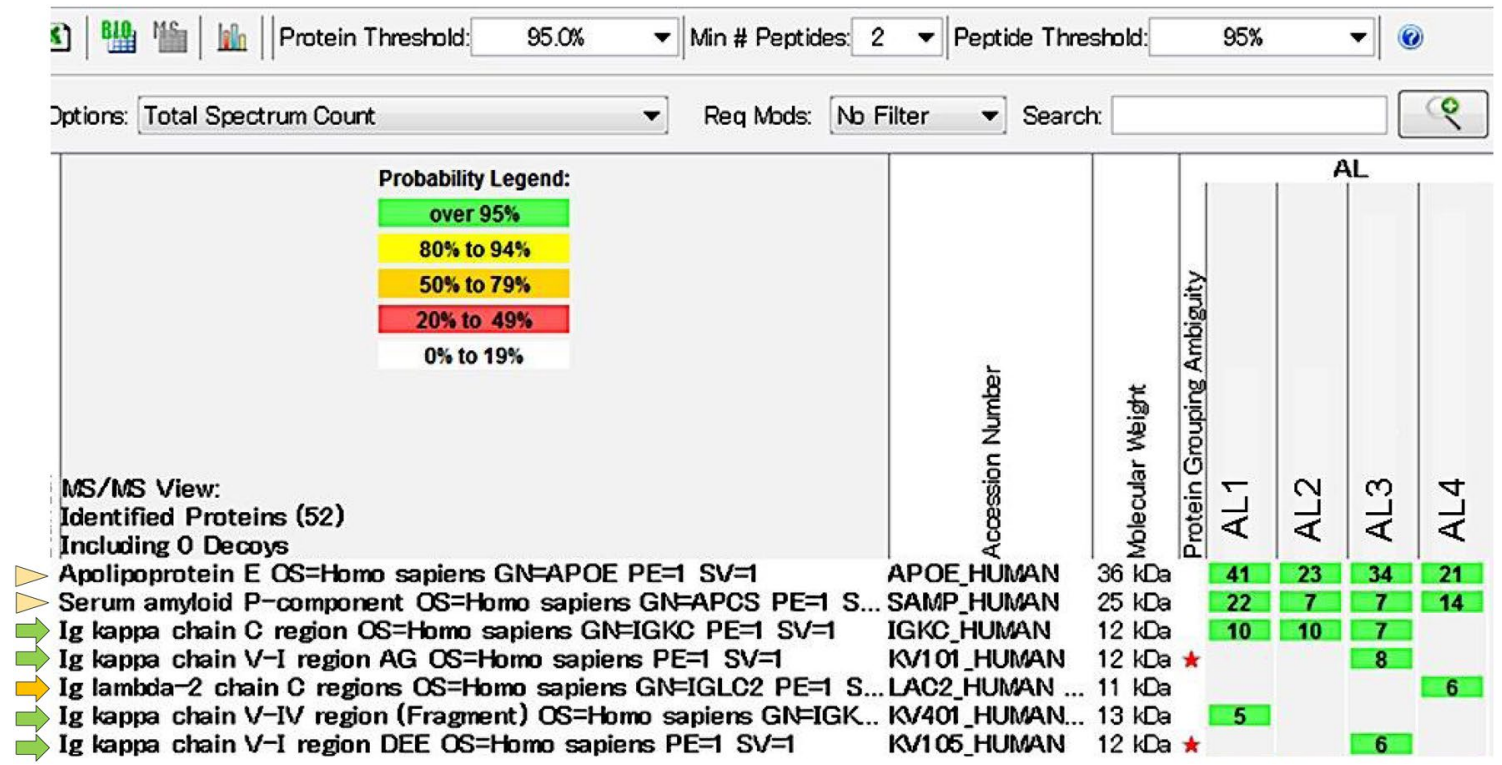

Fig. 7 Results of the LCMS/MS analysis using the Scaffold database in AL amyloidosis. Results of the LCMS/MS analysis of the 4 $\mathrm{AL}$ amyloidosis cases showed that the amyloid protein (single $\mathrm{k}$ or $\lambda$ light chain: green or yellow color arrows) was detected more than minimum spectra 4 level in the FFPE specimens, in conjunction with the amyloidogenic proteins (serum amyloid $\mathrm{P}$ and apolipoprotein $\mathrm{E}$ : orange color arrowhead). Result of the LCMS/MS for the AL1 cases

$[8,9]$. In our study, we found that 10 dissected glomeruli from 5- $\mu$ m-thick FFPE sections were necessary to be able to detect amyloid proteins in 10 out of 12 AL amyloidosis cases and in all 10 AA amyloidosis cases. In addition, showed there was a large spectra number of the $\mathrm{k}$ chain, similar to that found in the IF study. For the AL2 case, although the monoclonal immunoglobulin in the serum and urine could not be detected by IEP, LCMS/MS demonstrated the presence of the $\mathrm{k}$ light chain as a 10 spectra of the Ig k C region. For the 2 other AL cases (AL3 and AL4), there was only one immunoglobulin light chain that matched the findings of the IEP study

FFPE tissues were considerably more suitable than frozen tissues for the LCMS/MS analysis, as there were larger spectra numbers for the amyloid and amyloidogenic proteins in samples from FFPE tissues versus the frozen tissues. In 
addition, cytoskeletal proteins such as keratin, actin, and vimentin were higher in FFPE tissues than those in frozen tissues, indicating that the protein could be preserved by formalin fixation for a long time. It is well known that formalin fixation serves to stabilize the cellular and morphological details of cells. In DNA and mRNA extraction from glomerulus using LMD, the DNA volume is better from frozen tissues than FFPE tissues and may be associated with the degradation and breakage of DNA and mRNA by formalin fixation $[10,11]$. However, the proteins after formalin fixation can be preserved for a long time and can be analyzed by LCMS/MS, because the majority of the primary sequence of proteins may be protected from formalin fixation and many unmodified peptides can be recovered by digestion and readily identified by LCMS/MS [12]. In the present study, our frozen samples of amyloidosis cases were saved for more than 10 years. We considered the possibility that frozen samples could digest themselves even in a deep freezer in our department. The use of this technique therefore is advantageous, as this means that only small samples are required for the analysis, and FFPE tissues can be kept for a long time. When it is not possible to detect amyloid protein using 10 glomeruli from 5- $\mu \mathrm{m}$-thick FFPE sections, we should dissect additional glomeruli needed for the LCMS/MS analysis, because we found that the spectra numbers for the amyloid protein increased in accordance with the number of dissected glomeruli.

We subsequently examined the amount of trypsin and DDT required for the proteolytic procedures. During the course of the proteolysis, trypsin is the most common enzyme used for the procedure, due to its ability to only hydrolyze the C-terminal side of arginine or lysine [13]. DTT is a powerful reducing agent that is able to cleave the disulfide bonds among amyloid deposits, thereby preventing intermolecular and intermolecular disulfide formation in cysteines [14]. Our results showed that, even if the amount of trypsin $(0.1 \mathrm{mg} / \mathrm{mL})$ was increased from 3 to $20 \mu \mathrm{L}$ or if DTT was added to the AL amyloidosis samples for the LCMS/MS analysis, the mass spectra number for immunoglobulin light chain did not increase. The highest spectra for immunoglobulin $\kappa$ or $\lambda$ was noted in the samples with $3 \mu \mathrm{L}$ $(0.1 \mathrm{mg} / \mathrm{mL})$ of trypsin without DDT. In addition, the spectra of serum amyloid A in the samples with $3 \mu \mathrm{L}(0.1 \mathrm{mg} /$ $\mathrm{mL}$ ) of trypsin, while under the same conditions as that for $\mathrm{AL}$ amyloidosis, showed there were more than 4 spectra in all 4 of the AA amyloidosis cases. Thus, we considered that $3 \mu \mathrm{L}(0.1 \mathrm{mg} / \mathrm{mL})$ of trypsin might be sufficient for the digestion of AL and AA amyloidosis samples for LCMS/ MS analysis.

Interestingly, our results showed that the spectra numbers of the amyloid proteins determined by the LCMS/MS analysis were not correlated with the degree of the amyloid deposition rate. This may suggest that the extraction rate of the amyloid protein from the FFPE tissues differs in accordance with each of the amyloid proteins. In fact, when using our method, less than 4 spectra were evident in 2 out of 12 AL amyloidosis cases. Low spectra numbers were also noted in the $\mathrm{AL}$ amyloidosis versus the AA amyloidosis cases, even though the amyloid deposition rate was higher in the AA amyloidosis compared to the AL amyloidosis. While at least 31 amyloid proteins have been recently reported to be associated with amyloidosis, the optimal proteolytic conditions for the trypsin and DDT may differ for each of the amyloid proteins. If amyloid protein cannot be detected in samples with $3 \mu \mathrm{l}(0.1 \mathrm{mg} / \mathrm{mL})$ of trypsin, further examinations of the amyloid protein proteolysis will need to be undertaken to determine the optimal conditions for each of these amyloid proteins.

While IF and IHC studies remain the most widely used technique for detecting the amyloid protein, the results are sometimes inconclusive. Serum amyloid A, which is associated with chronic immune activation such as rheumatoid arthritis, has been reported to have a selective affinity for immunoglobulin components in the serum [2]. This could be associated with the nonspecific positive staining that is observed in some of the IF and IHC studies. Indeed, inconclusive positive staining of immunoglobulins and/or the absence of any amyloid protein staining was evident in the IF in the present study. It has been previously reported that in some cases, the monoclonal deposition of IgG $\kappa$ in glomeruli can be masked [15]. Although Hoshii et al. generated new antibodies against the immunoglobulin light chain constant and variable region in AL amyloid [16, 17], this technique is difficult to use in routine pathological studies. However, the use of the LCMS/MS proteomic analysis can compensate for these limitations in the detection of amyloid protein that occur when using these routine pathological techniques.

LCMS/MS can be used to identify not only well-known amyloid proteins, but also unknown amyloid proteins. Previous studies have shown that the LCMS/MS analysis can be used to detect these amyloid proteins in familial and hereditary amyloidosis, such as transthyretin (ATTR), leukocyte chemotactic factor 2 (LECT2), fibrinogen A $\alpha$ chain, and apolipoproteinA-I, A-II, and A-IV, which are otherwise difficult to diagnose when using routine pathological techniques [18-20]. Moreover, the LCMS/MS proteomic analysis has also been recently utilized to define the glomerular proteomic profile of various types of glomerulonephritis [21, 22].

Although LCMS/MS can detect amyloid protein and is superior as compared to classical pathological technique, its accuracy is less than $100 \%$ [7, 23-25]. This is thought to be related to several limitations that are associated with LCMS/ MS. The analysis of LSMS/MS can indicate the proteomic profile of tissues; however, there may be difficulty in finding which protein is associated with the amyloid protein, when 
it is an unknoun amyloid protein. In addition, identification is dependent on enzyme cutting sites and informatics, and the procedure might be less sensitive for low abundance proteins. There may also be issues with insufficient tissue volume due to problems during the biopsy [26].

\section{Conclusion}

LCMS/MS analysis of 10 dissected glomeruli using LMD from 5- $\mu \mathrm{m}$-thick FFPE sections with trypsin $3 \mu \mathrm{l}(0.1 \mathrm{mg} /$ $\mathrm{mL}$ ) without DDT was able to detect amyloid and amyloidogenic proteins in AA and AL amyloidosis. LCMS/MS can detect amyloid protein that is inconclusively stained by the IF and IHC techniques.

Acknowledgements We express special thanks to Mr Takashi Arai, Ms. Mitue Kataoka, Ms. Kyoko Wakamatsu, and Ms. Arimi Ishikawa for the expert technical assistance.

Funding This study was supported in part by Grants-in-Aid for Scientific Research from the Japan Society for the Promotion of Science; (C) no. 17K09717 (AK).

\section{Compliance with ethical standards}

Conflict of interest The authors have declared that no conflict of interest exists.

Ethical statement The protocol of this study was approved by the Clinical Study Ethics Review Board of Nippon Medical School. This study was conducted according to the principles of the Declaration of Helsinki and Istanbul.

Informed consent Prior to the study, informed consent was obtained from all patients (Clinical Study Ethics Review Board of Nippon Medical School, approval no. 28-12-679).

Open Access This article is distributed under the terms of the Creative Commons Attribution 4.0 International License (http://creativecommons.org/licenses/by/4.0/), which permits unrestricted use, distribution, and reproduction in any medium, provided you give appropriate credit to the original author(s) and the source, provide a link to the Creative Commons license, and indicate if changes were made.

\section{References}

1. Merlini G, Bellotti V. Molecular mechanisms of amyloidosis. N Engl J Med. 2003;349(6):583-96.

2. Satoskar AA, Burdge K, Cowden DJ, Nadasdy GM, Hebert LA, Nadasdy T. Typing of amyloidosis in renal biopsies: diagnostic pitfalls. Arch Pathol Lab Med. 2007;131(6):917-22.

3. Picken MM. Immunoglobulin light and heavy chain amyloidosis AL/AH: renal pathology and differential diagnosis. Contrib Nephrol. 2007;153:135-55.

4. Picken MM, Jerrera GA. The burden of "sticky" amyloid:typing challenges. Arch Pathol Lab Med. 2007;131(6):850-51.
5. Sethi S, Theis JD, Leung N, Dispenzieri A, Nasr SH, Fidler $\mathrm{ME}$, et al. Mass spectrometry-based proteomic diagnosis of renal immunoglobulin heavy chain amyloidosis. Clin J Am Soc Nephrol. 2010;5(12):2180-7.

6. Sethi S, Vrana JA, Theis JD, Leung N, Sethi A, Nasr SH, et al. Laser microdissection and mass spectrometry-based proteomics aids the diagnosis and typing of renal amyloidosis. Kidney Int. 2012;82(2):226-34.

7. Tasaki M, Obayashi K, Ueda M, Ando Y. Amyloid typing from formalin fixed paraffin-embedded tissue using LMD-LC-MS/ MS system. Rinsho Byori. 2014;62(3):291-6.

8. Sethi S, Gamez JD, Vrana JA, Theis JD, Bergen HR, Zipfel PF, et al. Glomeruli of dense deposit disease contain components of the alternative and terminal complement pathway. Kidney Int. 2009;75(9):952-60.

9. Vrana JA, Gamez JD, Madden BJ, Theis JD, Bergen HR, Dogan A. Classification of amyloidosis by laser microdissection and mass spectrometry-based proteomic analysis in clinical biopsy specimens. Blood. 2009;114(24):4957-9.

10. Koshiba M, Ogawa K, Hamazaki S, Sugiyama T, Ogawa O, Kitajima T. The effect of formalin fixation on DNA and the extraction of high-molecular-weight DNA from fixed and embedded tissues. Pathol Res Pract. 1993;189(1):66-72.

11. Groelz D, Sobin L, Branton P, Compton C, Wyrich R, Rainen L. Non-formalin fixative versus formalin-fixed tissue: a comparison of histology and RNA quality. Exp Mol Pathol. 2013;94(1):188-94.

12. Hood BL, Conrads TP, Veenstra TD. Mass spectrometric analysis of formalin-fixed paraffin-embedded tissue: unlocking the proteome within. Proteomics. 2006;6(14):4106-14.

13. Levin M, Franklin EC, Frangione B, Pras M. The amino acid sequence of a major nonimmunoglobulin component of some amyloid fibrils. J Clin Invest. 1972;51(10):2773-6.

14. Hong DP, Gozu M, Hasegawa K, Naiki H, Goto Y. Conformation of beta 2-microglobulin amyloid fibrils analyzed by reduction of the disulfide bond. J Biol Chem. 2002;277(24):21554-60.

15. Larsen CP, Ambuzs JM, Bonsib SM, Boils CL, Cossey LN, Messias NC, et al. Membranous-like glomerulopathy with masked IgG kappa deposits. Kidney Int. 2014;86(1):154-61.

16. Hoshii Y, Setoguchi M, Iwata T, Ueda J, Cui D, Kawano H, et al. Useful polyclonal antibodies against synthetic peptides corresponding to immunoglobulin light chain constant region for immunohistochemical detection of immunoglobulin light chain amyloidosis. Pathol Int. 2001;51(4):264-70.

17. Hoshii Y, Kiyama M, Cui D, Kawano H, Ishihara T. Immunohistochemical study of immunoglobulin light chain amyloidosis with antibodies to the immunoglobulin light chain variable region. Pathol Int. 2006;56(6):324-30.

18. Sethi S, Vrana JA, Theis JD, Dogan A. Mass spectrometry based proteomics in the diagnosis of kidney disease. Curr Opin Nephrol Hypertens. 2013;22(3):273-80.

19. Paueksakon P, Fogo AB, Sethi S. Leukocyte chemotactic factor 2 amyloidosis cannot be reliably diagnosed by immunohistochemical staining. Hum Pathol. 2014;45(7):1445-50.

20. Dasari S, Amin MS, Kurtin PJ, Vrana JA, Theis JD, Grogg KL, et al. Clinical, biopsy, and mass spectrometry characteristics of renal apolipoprotein A-IV amyloidosis. Kidney Int. 2016;90(3):658-64.

21. Sethi S, Fervenza FC, Zhang Y, Zand L, Vrana JA, Nasr SH, et al. C3 glomerulonephritis: clinicopathological findings, complement abnormalities, glomerular proteomic profile, treatment, and follow-up. Kidney Int. 2012;82(4):465-73.

22. Sethi S, Zand L, De Vriese AS, Specks U, Vrana JA, Kanwar S, et al. Complement activation in pauci-immune necrotizing and crescentic glomerulonephritis: results of a proteomic analysis. Nephrol Dial Transpl. 2017;32:i139-i45. 
23. Mollee P, Boros S, Loo D, Ruelcke JE, Lakis VA, Cao KL, et al. Implementation and evaluation of amyloidosis subtyping by laser-capture microdissection and tandem mass spectrometry. Clin Proteom. 2016;13:30.

24. Motwani SS, Herlitz L, Monga D, Jhaveri KD, Lam AQ. Forum ASoNO-N. Paraprotein-related kidney disease: glomerular diseases associated with paraproteinemias. Clin J Am Soc Nephrol. 2016;11(12):2260-72.
25. Said SM, Reynolds C, Jimenez RE, Chen B, Vrana JA, Theis JD, et al. Amyloidosis of the breast: predominantly AL type and over half have concurrent breast hematologic disorders. Mod Pathol. 2013;26(2):232-8.

26. Picken MM. Proteomics and mass spectrometry in the diagnosis of renal amyloidosis. Clin Kidney J. 2015; 8:665-72. 